\title{
Turning the oil tanker: a novel approach to shifting perspectives in medical practice
}

This article was published in the following Dove Press journal:

Advances in Medical Education and Practice

\section{Lasith Ranasinghe ${ }^{\prime}$ \\ Frank JMF Dor ${ }^{2-4}$ \\ Paul Herbert ${ }^{2-4}$}

'Faculty of Medicine, Imperial College London, London, UK; ${ }^{2}$ Imperial College Renal and Transplant Unit, Hammersmith Hospital, London, UK; ${ }^{3}$ Faculty of Medicine, Imperial College Healthcare NHS Trust, London, UK; ${ }^{4}$ Department of Surgery and Cancer, Imperial College, London, UK
Correspondence: Lasith Ranasinghe 9I Brecon Road, ammersmith, W6 8PXH, London, UK

Email IprII4@ic.ac.uk

\begin{abstract}
Although health care is encouraged to follow an evidence-based approach, there are perceived instances where suboptimal practice persists in the presence of better options due to an inherent resistance to change within many health care systems. To continue striving for clinical excellence, it is important to identify deficient practices and make appropriate corrections by implementing new and improved techniques and treatments. Bringing about change, however, tends to be a long, arduous process consisting of several small and successive deviations from the norm, analogous to "turning the oil tanker". Analyzing the methods employed by successful health care innovators has allowed the development of a "three-pronged" approach to overcoming resistance to change: 1) a determined opinion leader with a network or like-minded opinion leaders; 2) the presentation of hard evidence with adequate praise for current practice and the generation of clearly worded, specific guidelines; and 3) the use of simple reminders and continuous analysis of outcomes. Employing this three-pronged approach could lead to faster and more successful implementation of change within the health care system.
\end{abstract}

Keywords: health care innovation, resistance to change, implementing change, evidencebased medicine

\section{Introduction}

Modern medicine prides itself on an "evidence-based" approach. This involves responding to research findings and changing practice accordingly with the overarching aim of improving outcomes. ${ }^{1}$ In retrospect, it is easy to apply the tag of "evidence-based medicine" to many key changes in medical practice which appeared to cause an abrupt change in outcomes such as the introduction of antibiotics and vaccinations. However, resistance to change amongst doctors and organizations must be overcome to bring about major change. ${ }^{2}$ This phenomenon can be seen on a smaller, albeit important, scale when looking at differences within the UK in clinical guidelines for managing certain conditions. The establishment of GIRFT (Getting It Right First Time) in 2014, an organization that aims to identify discrepancies in practice and promote the adoption of evidencebased approaches, highlights the importance of this issue. ${ }^{3}$ Bringing about change tends to be a painstaking process consisting of a series of small deviations from the norm, analogous to "turning the oil tanker". However, by studying the efforts of successful innovators, the strengths of their approaches can be analyzed and assembled to generate a novel approach to shifting perspectives in medical practice.

\section{Reasons for resistance to change}

Perhaps the greatest barrier to change in health care occurs at an organizational level. The culture of innovation can, of course, vary between hospitals, organizations and, 
in some cases, governments. Quite often, organizations will view innovators as "troublemakers" or "radicals". 4 These attitudes may stem the progress of those proposing a change in practice, and discourage innovative thinking. Similarly, Sheard et al (2017) in their study of successful health care innovators reported that many study subjects commented that unwritten social conventions within the medical hierarchy imply that a doctor should reach a certain level of seniority before "rocking the boat" by proposing a change in practice. Consequently, innovators with revolutionary ideas may feel obliged to keep their ideas to themselves until they feel that going against the tide will not detriment their status. We propose that having an established protocol for proposing an innovation which would include signposting to relevant seniors with an interest in innovation and experience in the field could facilitate successful implementation.

Attitudes aside, the pace at which innovations are processed and considered for implementation by organizations, such as the NHS, can be slow. ${ }^{5,6}$ Complex bureaucratic processes and a lack of responsiveness to enthusiastic innovators can discourage such innovators from pursuing their ideas further.

A lack of clinical leadership in promoting a change in practice is often a major contributor to failing to bring about change. ${ }^{7}$ In the UK, GMC Outcomes for Graduates implores young doctors to "apply scientific methods and approaches to medical research and integrate these with a range of sources of information used to make decisions for care", thereby promoting the practice of evidence-based medicine. ${ }^{8}$ However, the process of continuous professional development (CPD) and carrying out audits/appraisals has become an arduous tick-box exercise for many doctors as it is enforced by strict points-based criteria as opposed to encouraged. ${ }^{9}$ The research and design of an innovation may take a considerable amount of time and may not harvest many "points" via the regulatory bodies for CPD. The Royal College of Physicians guidelines for CPD only allow a maximum of 10 points (out of a minimum annual credit requirement of 50 points) for personal initiatives. $^{10}$

Methods such as the audit cycle are designed to encourage doctors to practice scientific method. In many cases, audit can be a gateway to innovation as it encourages critical analysis of deficiencies in clinical practice, and the implementation and evaluation of a potential solution. However, many pitfalls have also been widely documented. For example, soon after the national introduction of the audit cycle in clinical practice, Kogan and Redfern (1995) found that audit was hindered by a lack of time, under-funding and a lack of expertise whilst several other studies report that clinicians feel that audit "detracts from clinical work at the expense of patient care". ${ }^{11,12}$ Furthermore, many critics state that audit has led to few obvious benefits to patients. ${ }^{13}$ This notion has been supported by several studies including a review of 63 recommendations of audits by Prasad and Reddy (2004) which showed that only $28.5 \%$ of the recommendations were successfully implemented. $^{14}$

In summary, the literature suggests that backwards organizational cultures and rigid regulation and accreditation of continuing professional development, stifle creativity and impede the implementation of beneficial changes in practice.

\section{Past efforts to overcome resistance to change}

Several systematic reviews exploring the implementation of change in health care have suggested that effective strategies include reminders, patient-mediated interventions and opinion leaders, whilst less effective strategies include didactic lectures and printed teaching materials. $^{15,16}$ There is, however, some disagreement regarding the effectiveness of audit and feedback, thus highlighting the difficulty in evaluating methods which are inherently variable. ${ }^{17}$

A recurring theme in the literature regarding implementing change is the importance of a key opinion leader. ${ }^{18}$ Valued members of the health care team with sufficient peer and management support and an awareness of local organizational needs have been vital in pushing through changes in practice. Barnett et al (2011) conducted a study of successful pioneers in health care and identified four recurring themes: personal determination, brokering relationships and making connections, navigating organizational culture and using evidence to influence others. In particular, the use of hard data to promote an idea is particularly powerful and has been described as serving "a stabilising purpose for ideational claims". ${ }^{19}$ In addition, special attention should be paid to how the data is presented, as tailoring the delivery to the needs of the audience has also been described as an important factor contributing to the implementation of innovations. ${ }^{16}$ Delivering the 
data without paying due respect to the clinical excellence that is currently practiced by the audience and failing to mention how practice has improved thus far is likely to be met with more resistance. Although evidence-based medicine, as previously mentioned, is encouraged amongst doctors, they will, nonetheless, have a tendency to attribute considerable weight to their personal experiences. ${ }^{20}$ Although this is largely anecdotal and does not follow a well-defined evidencebase, the power of "experience-based medicine" should not be underestimated. This notion of experience-based medicine may be somewhat propagated by compulsory initiatives such as appraisals which promote reflection on personal practice without encouraging diligent attention to the wider scope of evidence-based medicine.

Guidelines have increasingly become more prominent in day-to-day medical practice. It has been shown that using concrete statements with more specifically described behaviors improved understanding and compliance with the guidelines. ${ }^{21}$ The merger of specific guidelines and regular reminders have been trialed to great effect in the past. For example, the use of a sticker in the shape of a breast with a bright orange dot placed on the charts of women over the age of 50 years helped achieve their aim of increasing referral rates for mammography across $61 \mathrm{GP}$ practices. ${ }^{22}$ Similarly, a $20 \%$ reduction in the rates of referral for X-rays of the lumbar spine and knee from 244 GP practices was achieved by placing a sticker with the specific guidelines for referral on the X-ray results of patients who had been referred. ${ }^{23}$ This highlights the powerful impact of simple reminders in encouraging the adoption of changes in practice.

Reviewing the literature shows that there are several themes that are common amongst success stories in the implementation of changes in practice. Strong opinion leaders with extensive networks, hard evidence with an audience-tailored delivery and regular reminders proved to be especially effective.

\section{A novel, three-pronged approach to overcoming resistance to change}

Considering the previously discussed features of successful implementation, a three-pronged approach can be used as a strategy to overcoming resistance. The implementation of pre-emptive renal transplantation (renal transplantation before starting dialysis as renal replacement therapy) will be used as an example to illustrate the strategy.

\section{Three Prongs:}

1. Determined opinion leader with a network of likeminded opinion leaders

2. Presentation of hard evidence with adequate praise for current practice and the generation of clearly worded, specific guidelines

3. Use of simple reminders and continuous analysis of outcomes

\section{Determined opinion leader}

The first step in the approach involves the establishment of a determined opinion leader who takes ownership of the project. The leader, however, cannot expect to make changes alone, irrespective of their seniority or status. A network of like-minded opinion leaders in every domain of health care that will be affected by the proposed change is necessary to disseminate the idea through the system and promote change. Salisbury et al (2015) described that "interventions designed in isolation can potentially lead to duplication, inefficiency and confusion". ${ }^{24}$ On a similar note, a report by the King's Fund highlighted how the health care system in the UK is compartmentalized into "service silos and geographical silos" with few formal mechanisms for disseminating information and knowledge across sites. ${ }^{6}$ This makes it more difficult to implement change across multiple sites, thereby confirming the importance of an extensive network of advocates for the proposed change spanning across trusts and deaneries. Whilst maintaining this team-based approach, oversight by a key individual, or a small group of individuals is still required as a lack of a clear point-of-contact may lead to social loafing and, ultimately, failure of the initiative.

The opinion leader should have specialist expertise in the field in question (eg, experienced renal transplant surgeon), such that their views are highly regarded. The opinion leader is responsible for establishing contact with other key figures throughout the health care systems (eg, other renal transplant surgeons, renal physicians, nurses, junior doctors, management staff, patient organisations, regional and national scientific groups and organizations) and create a team of opinion leaders who support the proposed change to conduct a quality improvement project. This support can be highlighted in the second step when the idea is delivered to the masses.

\section{Presentation of hard evidence}

The presentation would be best delivered as an easily digestible animation or slideshow. It should be tailored to the needs of the audience and address the priorities of all the 
stakeholders (eg, benefits to the patient, changes in doctors' workload, cost consideration for health care administrators, etc.).

The presentation should provide some historical context to the issue in question (eg, past advances in renal transplant protocol). It should begin by showing the beneficial impact of the last change in practice that was universally adopted by professionals in that field. This is designed to instil pride in the profession and commend the audience's contribution to improving practice thus far. Therefore, the novel idea will seem more like a natural next step in a continuum of advancing clinical practice rather than an abrupt and reckless diversion from the norm.

This should be followed by the presentation of clearly worded, specific guidelines for the proposed change. The data from the literature supporting the change in practice should be distilled and the key details should be presented. This should be followed by a projection, based on the data, of how the implemented change in practice will affect the organization over a certain time-period (eg, showing the improvement in patient outcomes, reduction in doctors' workload and reduction in cost to the organization if the guidelines for pre-emptive transplantation are followed for 5 years). A pragmatic stance should be taken when generating this projection (eg, if it is arbitrarily assumed that only $70 \%$ of doctors will follow the proposed guidelines, set the projection to account for $70 \%$ uptake thereby making it less idealistic and more realistic).

The presentation should also include information about the proponents of this change. Having several advocates at varying levels and roles within the health care network lends weight to the proposition and portrays the change as a team effort rather than the work of an isolated maverick.

The presentation should end with a summary of the benefits of the proposed change and reiteration of the new guidelines.

\section{Use of simple reminders}

Even with the agreement of health care professionals to implement a change in practice, it can often be difficult to achieve due to a tendency for clinicians to revert to their routine behaviors - a form of clinical "muscle memory". A simple reminder in the form of a sticker/tag (either electronic or paperbased) with a summary of the new guidelines (eg, for preemptive renal transplantation) should be stuck on the notes of patients who have been referred for pre-emptive renal transplantation to provide positive-reinforcement for that behavior.
In addition, once the change has been agreed upon, data should continue to be collected to show ongoing uptake rates of the new idea and its benefits. As a patient-centered approach is a dominant principle of modern day medical practice, ${ }^{25}$ data should also be collected from patients who have been subject to the new guidelines. This is a compelling way of consolidating the behavior amongst doctors and disseminating the idea amongst patients.

\section{Conclusion}

The implementation of new, evidence-based ideas has lagged behind the pace at which such innovations are generated. The sensitive nature of medical practice means that it is important to critically analyze all proposed changes and thoroughly assess new ideas to ensure that they will improve health care. However, resistance to change often stems from social taboos, lack of leadership and misguided delivery of the innovation. Distilling and analyzing past campaigns for changes in practice suggests that a "Three-pronged approach" formulated by the amalgamation of three complementary, and proven to be successful, strategies, may result in improved uptake of novel ideas.

\section{Disclosure}

Frank JMF Dor reports personal fees from Astellas, Chiesi, Sandoz, and TEVA, outside the submitted work, and reports no other conflicts of interest. Paul Herbert and Lasith Ranasinghe report no conflicts of interest in regard to this work.

\section{References}

1. Evidence-Based Medicine Working Group. Evidence-based medicine. A new approach to teaching the practice of medicine. JAMA. 1992;268 (17):2420-2425. doi:10.1001/jama.1992.03490170092032

2. Li Bassi G, Ranzani O, Torres A. Systematic implementation of evidence-based guidelines in intensive care medicine. Crit Care Med. 2013;41(1):329-331. doi:10.1097/CCM.0b013e318270e234

3. Getting it right first time - GIRFT [Internet] [updated November 19, 2018]. Available from: http://gettingitrightfirsttime.co.uk/. Accessed November 28, 2018

4. Sheard L, Jackson C, Lawton R. How is success achieved by individuals innovating for patient safety and quality in the NHS? BMC Health Serv Res. 2017;17:640. doi:10.1186/s12913-017-2589-1

5. Baxter R, Taylor N, Kellar I, Lawton R. Learning from positively deviant wards to improve patient safety: an observational study protocol. $B M J$ Open. 2015;5:E009650. doi:10.1136/bmjopen-2015-009650

6. Collins B. A doption and spread of innovation in the NHS [Internet]. Kingsfund.org.uk; 2018 [updated November 19, 2018]. Available from: https://www.kingsfund.org.uk/sites/default/files/2018-01/ Adoption_and_spread_of_innovation_NHS_0.pdf.

7. Caldwell DF, Chatman J, O'reily CA iii, Ormiston M, Lapiz M. Implementing strategic change in a health care system: the importance of leadership and change readiness. Health Care Manage Rev. 2008;33 (2):124-133. doi:10.1097/01.HMR.0000304501.82061.e0 
8. gmc.uk.org. [online]; 2018. Avialable from: https://www.gmc-uk. org/-/media/documents/dc11326-outcomes-for-graduates-2018_pdf75040796.pdf. Accessed November 14, 2018.

9. Schostak J, Davis M, Hanson J, Schostak J, Brown T. 'Effectiveness of continuing professional development' project: a summary of findings. Med Teach. 2010;32(7):586-592. doi:10.3109/0142159X.2010.489129

10. Guidelines for CPD diary [Internet]. RCP London. 2019 [updated 23 April 2019]. Available from: https://www.rcplondon.ac.uk/educationpractice/advice/guidelines-cpd-diary.

11. Kogan M, Redfern S. Making Use of Clinical Audit: A Guide to Practice in the Health Professions. Buckingham: Open University Press; 1995.

12. Smith HE, Russell GI, Frew AJ, et al. Medical audit: the differing perspectives of managers and clinicians. $J R$ Coll Physicians Lond. 1992;26:177-180.

13. Miles A, Bentley P, Polychronis A, Price N, Grey J. Clinical audit in the National Health Service: fact or fiction? J Eval Clin Pract. 1996;2:29-35. doi:10.1111/jep.1996.2.issue-1

14. Prasad K, Reddy K. Auditing the audit cycle: an open-ended evaluation. Clin Gov. 2004;9(2):110-114. doi:10.1108/14777270410536394

15. Davis DA, Thomson MA, Oxman AD, Haynes RB. Changing physician performance a systematic review of the effect of continuing medical education strategies. JAMA. 1995;274(9):700-705.

16. Bloom BS. Effects of continuing medical education on improving physician clinical care and patient health: a review of systematic reviews. Int J Technol Assess Health Care. 2005;21(3):380-385.

17. Grimshaw JM, Shirran L, Thomas R, Mowatt G, Fraser C. Changing provider behavior: an overview of systematic reviews of interventions. Med Care. 2001;39(8):112-145. doi:10.1097/00005650-200 108002-00002
18. Barnett J, Vasileiou K, Djemil F, Brooks L, Young T. Understanding innovators' experiences of barriers and facilitators in implementation and diffusion of healthcare service innovations: a qualitative study. BMC Health Serv Res. 2011;11:342. doi:10.1186/1472-6963-11-342

19. May C, Mort M, Williams T, Mair F, Gask L. Health technology assessment in its local context: studies in telehealthcare. Soc Sci Med. 2003;57:679-710. doi:10.1016/S0277-9536(02)00419-7

20. Koukoura O, Hajiioannou I. Exception, evidence, experience-based medicine: the evolution of medical practice and the Greek paradox. Med Teach. 2014;36(8):730-731. doi:10.3109/0142159X.2014. 909011

21. Ley P. Written communication. In: Baum A, Newman S, Weinman J, West R, McManus C, editors. Cambridge Handbook of Psychology, Health and Medicine. Cambridge: Cambride University Press; 1997:304-308.

22. Grady KE, Lemkau JP, Lee NR, Caddell C. Enhancing mammography referral in primary care. Prev Med. 1997;26:791-800. doi:10.1006/pmed.1997.0219

23. Eccles M, Steen N, Grimshaw J, Thomas L, Mcnamee P. Effect of audit and feedback, and reminder messages on primary-care radiology referrals: a randomised trial. Lancet. 2001;257(9266):14061409. doi:10.1016/S0140-6736(00)04564-5

24. Salisbury C, Thomas C, O'cathain A, et al. Telehealth in chronic disease: mixed-methods study to develop the TECH conceptual model for intervention design and evaluation. BMJ Open. 2015;5: e006448. doi:10.1136/bmjopen-2014-006448

25. Stewart M. Towards a global definition of patient centred care. $B M J$. 2001;322(7284):444-445. doi:10.1136/bmj.322.7284.444
Advances in Medical Education and Practice

\section{Publish your work in this journal}

Advances in Medical Education and Practice is an international, peerreviewed, open access journal that aims to present and publish research on Medical Education covering medical, dental, nursing and allied health care professional education. The journal covers undergraduate education, postgraduate training and continuing medical education including emerging trends and innovative models linking education, research, and health care services. The manuscript management system is completely online and includes a very quick and fair peer-review system. Visit http://www.dovepress.com/testimonials.php to read real quotes from published authors. 